\title{
Holographic Wilson loops, Hamilton-Jacobi equation, and regularizations
}

\author{
Diego Pontello ${ }^{1, *}$ and Roberto Trinchero ${ }^{1,2, \dagger}$ \\ ${ }^{1}$ Instituto Balseiro, Centro Atómico Bariloche, 8400 San Carlos de Bariloche, Argentina \\ ${ }^{2}$ CONICET, Rivadavia 1917, 1033 Capital Federal, Argentina
}

(Received 28 September 2015; published 4 April 2016)

\begin{abstract}
The minimal area for surfaces whose borders are rectangular and circular loops are calculated using the Hamilton-Jacobi (HJ) equation. This amounts to solving the HJ equation for the value of the minimal area, without calculating the shape of the corresponding surface. This is done for bulk geometries that are asymptotically anti-de Sitter (AdS). For the rectangular contour, the HJ equation, which is separable, can be solved exactly. For the circular contour an expansion in powers of the radius is implemented. The HJ approach naturally leads to a regularization which consists in locating the contour away from the border. The results are compared with the $\epsilon$-regularization which leaves the contour at the border and calculates the area of the corresponding minimal surface up to a diameter smaller than the one of the contour at the border. The results for the circular loop do not coincide if the expansion parameter is taken to be the radius of the contour at the border. It is shown that using this expansion parameter the $\epsilon$-regularization leads to incorrect results for certain solvable non-AdS cases. However, if the expansion parameter is taken to be the radius of the minimal surface whose area is computed, then the results coincide with the HJ scheme. This is traced back to the fact that in the HJ case the expansion parameter for the area of a minimal surface is intrinsic to the surface; however, the radius of the contour at the border is related to the way one chooses to regularize in the $\epsilon$-scheme the calculation of this area.
\end{abstract}

DOI: 10.1103/PhysRevD.93.075007

\section{INTRODUCTION}

The relation between large $N$ gauge theories and string theory [1] together with the AdS/CFT correspondence [2-5] has opened new insights into strongly interacting gauge theories. The application of these ideas to QCD has received significant attention since those breakthroughs. From the phenomenological point of view, the so-called AdS/QCD approach has produced very interesting results in spite of the strong assumptions involved in its formulation [6-11]. It seems important to further proceed investigating these ideas and refining the current understanding of a possible QCD gravity dual.

In the holographic approach, the vacuum expectation value of the Wilson loop is obtained by minimizing the Nambu-Goto (NG) action for a loop lying in the boundary space $[12,13]$. This is known to work in the strictly anti-de Sitter (AdS) case, i.e., for a conformal boundary field theory. In this work it is assumed that this procedure also works in the nonconformal-QCD case provided an adequate fivedimensional background metric is chosen. It is remarked that the vacuum expectation value of Wilson loops are phenomenologically relevant quantities directly connected with the condensates of the gauge theory [14-16]. In this respect, as shown in [16], it is worth remarking that there is a direct relation between the phenomenological values for the gauge

\footnotetext{
*diego.pontello@ib.edu.ar †trincher@cab.cnea.gov.ar
}

theory condensates and the coefficients in the expansion of the warp factor around the AdS case.

In this work the minimal area is computed by solving the Hamilton-Jacobi (HJ) equation. This approach has the advantage that the minimal area can be obtained without solving the equations of motion. It amounts to studying the variation of the minimal area under changes in the location and shape of the contour. This approach naturally leads to a regularization which consists in moving the contour into the bulk out of the border. This HJ regularization was also considered in [17] for the AdS case and in [18] in relation with UV divergences. In the former reference another regularization was also employed, which consists in locating the contour at the border but computing the area only up to a diameter smaller than that of the contour. This approach will be referred to as the $\epsilon$-scheme. It was shown that for the AdS case the results for smooth surfaces computed using both schemes coincide except with respect to zigzag symmetry. The HJ scheme respects this symmetry, but the $\epsilon$-scheme does not. In the present work, it is shown that for the non-AdS case, depending on the choice of expansion parameter, the results for the coefficients of the expansion in powers of the diameter of the circular contour of the NG action may not coincide for both schemes, even for regular surfaces. Furthermore, it is shown that choosing the radius of the loop at the border as the expansion parameter, the $\epsilon$-regularization scheme leads to wrong results for certain solvable non-AdS cases.

The features and results of this work are summarized as follows: 
(i) The HJ approach is employed for the calculation of minimal areas of rectangular and circular loops in asymptotically AdS spaces.

(ii) For the case of the rectangular loop the HJ equation is separable and can be solved exactly.

(iii) For the case of the circular loop an expansion of the NG on-shell action in powers of the radius of the loop is implemented. At each order the relevant differential equation is linear and solvable up to the calculation of an integral. For the cases where the warp factor in conformal coordinate $z$ is a pure power $z^{2 n}$, these integrals can be exactly calculated for any $n \geq-1$.

(iv) The HJ approach naturally leads to a regularization that consists in locating the loop contour away from the border. The divergences are removed by means of substracting to the regularized result and adequate number of derivatives with respect to the geometrical parameter characterizing the loop.

(v) The two regularizations considered in [17] are applied in this case. One of them is the one mentioned above that fits naturally in the HJ approach. The other one considers a minimal surface whose contour is at the border and computes the area of the surface up to a diameter smaller than the one of the contour at the border.

(vi) The results for the expansion coefficients of the NG on-shell action in powers of the radius are considered. For the circular loop, depending on the choice of expansion parameter, the results may not coincide for the two regularizations mentioned above. It is shown that the $\epsilon$-regularization, using the radius of the contour at the border as an expansion parameter, leads to incorrect results for certain solvable non-AdS cases. This is investigated in detail and has its origin in the use of the above mentioned regularization dependent expansion parameter in this scheme.

This paper is organized as follows. Section II defines the bulk metrics to be considered and recalls the NG action. Section III deals with the rectangular loop in the HJ approach. Section IV studies the circular loop in the HJ approach and the approximate solution of the HJ equation as a power series in the loop's radius. Section V deals with the substraction scheme and its explicit computation. Section VI compares both regularizations. The failure of the $\epsilon$-regularization for certain solvable non-AdS cases is explained, and the origin of this situation is considered. Section VII presents some concluding remarks. In addition two appendixes are included, one of them giving explicit expressions of the expansion coefficients mentioned above and the other showing the source of the differences between both regularization schemes.

\section{THE NAMBU-GOTO ACTION}

The distance to be considered has the following general form:

$$
\begin{aligned}
d s^{2} & =e^{2 A(z)}\left(d z^{2}+\eta_{i j} d x^{i} d x^{j}\right) \\
& =G_{\mu \nu} d x^{\mu} d x^{\nu} \quad \mu, \nu=1, \ldots, d+1 .
\end{aligned}
$$

It is defined by a metric with no dependence on the boundary coordinates, which therefore preserves the boundary space Poincaré invariance. This should be the case if only vacuum properties are considered. The form of the warp factor $A(z)$ to be considered is

$$
A(z)=-\ln \left(\frac{z}{L}\right)+f(z)
$$

where $f(z)$ is a dimensionless function. In this work $f(z)$ is taken to be a series in even ${ }^{1}$ powers of $z$, i.e.,

$$
f(z)=\sum_{k=1} \alpha_{2 k} z^{2 k}
$$

The case $f(z)=0$ corresponds to the AdS metric. This deviation from the AdS case could be produced by a bulk gravity theory including matter fields. Possible candidates for these bulk gravity theories have been considered in $[19,20]$.

The area of a surface embedded in this space is given by the NG action,

$$
S_{\mathrm{NG}}=\frac{1}{2 \pi \alpha^{\prime}} \int d^{2} \sigma \sqrt{g}
$$

where $g$ is the determinant of the induced metric on the surface, which is given by

$$
g_{a b}=G_{\mu \nu} \partial_{a} x^{\mu} \partial_{b} x^{\nu}
$$

where $x^{\mu}(a, b)$ are the coordinates of the surface embedded in the ambient $(d+1)$-dimensional space. The indices $a, b$ refer to coordinates on the surface.

\section{RECTANGULAR LOOP}

The surface contoured by this loop is described by the following embedding:

$$
\begin{aligned}
x^{1} & =t, \quad t \in\left[-\frac{T}{2}, \frac{T}{2}\right], \\
x^{i} & =x, \quad x \in[-a, a], \\
x^{k} & =0, \quad \forall k \neq i, \\
x^{5} & =z=z(x) .
\end{aligned}
$$

The determinant of the induced metric,

\footnotetext{
${ }^{1}$ Restricting to even powers implies that no odd dimensional condensates will appear [16]. The motivation for this requirement is that this is the case in QCD where no odd dimensional condensates appear.
} 

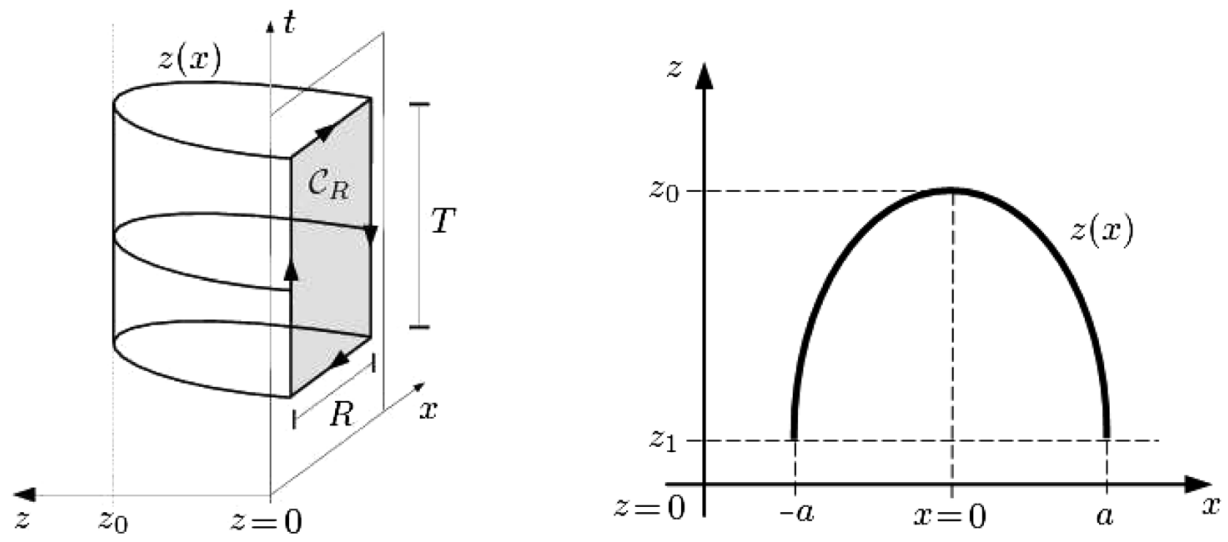

FIG. 1. (a) The rectangular loop is located at $z=0$. The corresponding world sheet $z(x)$ lives in the bulk. For $T \rightarrow \infty$, the world sheet parametrization $z(x)$ is $t$ independent. (b) A world sheet section for fixed $t$. In this case the contour is located at a value $z_{1}$. This value can be sent to zero after substraction.

$$
g_{a b}=G_{\mu \nu} \partial_{a} x^{\mu} \partial_{b} x^{\nu} \quad(a, b=t, x),
$$

is given by

$$
\operatorname{det}\left(g_{a b}\right)=\left[1+z^{\prime}(x)^{2}\right] \mathrm{e}^{4 A(z)}
$$

where in the last equality translation and reflection symmetry has been employed. The geometrical setting given above is described in Fig. 1.

As described in Fig. 2, the loop is located at a value $z_{1}$ of the coordinate orthogonal to the border; in addition, the corresponding minimal surface is required to be regular at the origin. Therefore the boundary conditions for the minimal surface are

$$
z(a)=z_{1}, \quad z^{\prime}(0)=0
$$

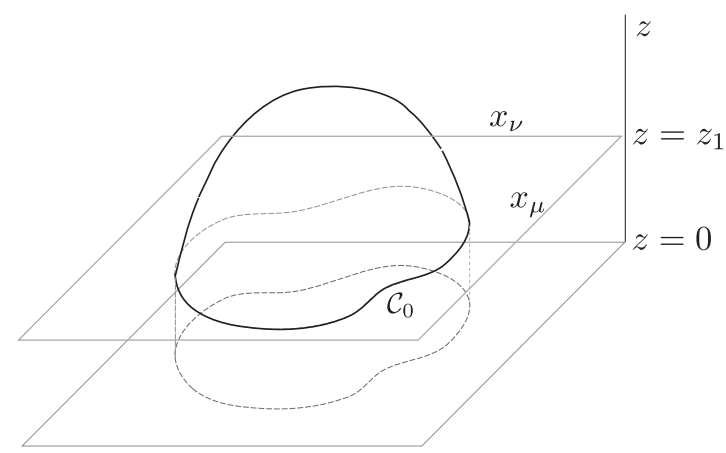

FIG. 2. A loop located at $z=z_{1}$, and the corresponding world sheet.
The potential between static quarks can be obtained from the NG action as follows:

$V_{\bar{q} q}(R)=\lim _{T \rightarrow+\infty} \frac{S_{N G}}{T}=\frac{1}{\pi \alpha^{\prime}} \int_{0}^{a} d x \mathrm{e}^{2 A(x)} \sqrt{1+z^{\prime}(x)^{2}}$,

where $R=2 a$ is the interquark separation.

\section{A. Hamilton-Jacobi approach}

With the boundary conditions mentioned above, the on-shell NG action is a function of the interquark separation $a$ and $z_{1}$, the location of the loop, i.e.,

$$
S_{\mathrm{NG}}=S_{\mathrm{NG}}\left(a, z_{1}\right),
$$

and the corresponding Hamilton-Jacobi equation is given by

$$
\frac{\partial S_{\mathrm{NG}}\left(a, z_{1}\right)}{\partial a}+H\left(z_{1}, \frac{\partial S_{\mathrm{NG}}\left(a, z_{1}\right)}{\partial z_{1}}, a\right)=0,
$$

where $H=H(z, p, x)$ is the Hamiltonian, $p$ the canonical conjugate momenta to $z$, and $x$ the coordinate along the spatial dimension of the loop. To make the calculations easier, it is helpful to neglect the multiplicative factor $\frac{T}{\pi \alpha^{\prime}}$ in (3.1) and reintroduce it in the final expression. Standard methods lead to

$$
\begin{aligned}
H(z, p, x) & =p z^{\prime}(z, p, x)-L\left(z, z^{\prime}(z, p, x), x\right) \\
& =-\frac{\mathrm{e}^{2 A(z)}}{\sqrt{1+z^{\prime 2}}}=-\sqrt{\mathrm{e}^{4 A(z)}-p^{2}}
\end{aligned}
$$

leading to the following form of the $\mathrm{HJ}$ equation:

$$
\frac{\partial S_{\mathrm{NG}}\left(a, z_{1}\right)}{\partial a}-\sqrt{\mathrm{e}^{4 A\left(z_{1}\right)}-\left[\frac{\partial S_{\mathrm{NG}}\left(a, z_{1}\right)}{\partial z_{1}}\right]^{2}}=0 .
$$


In this case, since the Lagrangian does not depend on the coordinate $x$, the Hamiltonian is a constant of motion $E$; thus,

$$
\frac{\partial S_{\mathrm{NG}}\left(a, z_{1}\right)}{\partial a}=\sqrt{\mathrm{e}^{4 A\left(z_{1}\right)}-\left[\frac{\partial S_{\mathrm{NG}}\left(a, z_{1}\right)}{\partial z_{1}}\right]^{2}}=-E,
$$

and the value of $E$ can be obtained from (3.3) as follows:

$$
E=-\frac{\mathrm{e}^{2 A(z)}}{\sqrt{1+z^{\prime 2}}}=-\mathrm{e}^{2 A\left(z_{0}\right)}
$$

where $z_{0}$ is the maximum value of the coordinate $z$ attained by the minimal surface, which is therefore such that

$$
z_{0}=z(0), \quad z^{\prime}(0)=0
$$

An expression for $z_{0}$ as a function of $a$ and $z_{1}$ can be obtained by means of

$$
\begin{aligned}
a & =\int_{0}^{a} d x=\int_{z(0)}^{z(a)} \frac{d x}{d z} d z \\
& =\int_{z_{0}}^{z_{1}} \frac{1}{z^{\prime}} d z=\int_{z_{1}}^{z_{0}} \frac{\mathrm{e}^{2 A\left(z_{0}\right)}}{\sqrt{\mathrm{e}^{4 A(z)}-\mathrm{e}^{4 A\left(z_{0}\right)}}} d z .
\end{aligned}
$$

Having a constant of motion, a solution by separation of variables is possible,

$$
S_{\mathrm{NG}}\left(a, z_{1}\right)=A(a)+Z\left(z_{1}\right),
$$

and replacing in (3.4) gives

$$
\begin{aligned}
A^{\prime}(a) & =-E \\
Z^{\prime}\left(z_{1}\right) & = \pm \sqrt{\mathrm{e}^{4 A\left(z_{1}\right)}-E^{2}} .
\end{aligned}
$$

The general solution to these equations is ${ }^{2}$

$$
\begin{aligned}
A(a) & =-E \cdot a+A_{0}, \\
Z\left(z_{1}\right) & =-\int_{z_{\text {inf }}}^{z_{1}} \sqrt{\mathrm{e}^{4 A(z)}-E^{2}} d z
\end{aligned}
$$

where the integration constants $A_{0}$ and $z_{\text {inf }}$ have to be determined by choosing adequate boundary conditions. The following boundary condition is adopted:

$$
\lim _{a \rightarrow 0} S_{\mathrm{NG}}\left(a, z_{1}\right)=0, \quad \forall z_{1} .
$$

This condition is satisfied by the following solution:

\footnotetext{
${ }^{2}$ In the second equation below, the minus sign has been chosen. This choice corresponds to a minimal surface that extends from the border $z=0$ to greater values of $z$.
}

$$
\begin{aligned}
A(a) & =-E \cdot a \\
Z\left(z_{1}\right) & =-\int_{z_{0}}^{z_{1}} \sqrt{\mathrm{e}^{4 A(z)}-E^{2}} d z .
\end{aligned}
$$

Noting that $\lim _{a \rightarrow 0} z_{0}=z_{1}$ shows that the required boundary condition (3.7) is fulfilled.

Replacing $S_{\mathrm{NG}}\left(a, z_{1}\right)$ in (3.2), the interquark potential is given by

$V_{\bar{q} q}(R)=\frac{1}{2 \pi \alpha^{\prime}}\left[R \mathrm{e}^{2 A\left(z_{0}\right)}+2 \int_{z_{1}}^{z_{0}} \sqrt{\mathrm{e}^{4 A(z)}-\mathrm{e}^{4 A\left(z_{0}\right)}} d z\right]$,

which coincides with the results in [21]. In order to express this potential in terms of $a$ and $z_{1}$, Eq. (3.5) can be employed to obtain $z_{0}$ as a function of $a$ and $z_{1}$. In the AdS case $A(z)=-\ln \left(\frac{z}{L}\right)$, the integrals appearing in (3.5) and (3.8) are elliptic and can be evaluated to give expressions in terms of the hypergeometric function. In the general case, near the border, i.e., for $z_{1} \rightarrow 0$, the integrals can be evaluated up to terms proportional to positive powers of $z_{1}$, leading to

$$
\begin{aligned}
V_{\bar{q} q}(R) & =\frac{L^{2}}{2 \pi \alpha^{\prime}}\left[R e^{2 A\left(z_{0}\right)}+\frac{\sqrt{\pi} \Gamma\left(-\frac{1}{4}\right)}{4 z_{0} \Gamma\left(\frac{5}{4}\right)}+\frac{2}{z_{1}}\right]+\mathcal{O}\left(z_{1}\right), \\
a & =\frac{\sqrt{\pi} z_{0} \Gamma\left(\frac{3}{4}\right)}{\Gamma\left(\frac{1}{4}\right)}+\mathcal{O}\left(z_{1}^{3}\right)
\end{aligned}
$$

which clearly shows that there is a divergence for $z_{1} \rightarrow 0$. This happens also in the non-AdS case and is related to the divergence of the metric near the border. A substraction procedure should be employed to obtain a finite value. This substraction will be discussed in Sec. V.

\section{CIRCULAR LOOP}

The surface contoured by the circular loop is described by the following embedding:

$$
\begin{aligned}
& x^{1}=0, \\
& x^{\sigma}=0 \quad \forall \sigma \neq \mu, \nu, \\
& x^{\mu}=r \cos (\varphi), \\
& x^{\nu}=r \sin (\varphi), \\
& x^{5}=z=z(r), \quad 0 \leq \varphi \leq 2 \pi, \quad 0 \leq r \leq a .
\end{aligned}
$$

It should be noted that the coordinate $z$ has been taken to depend only on $r$, due to the rotational symmetry of the contour and the metric. The corresponding geometrical setting is shown in Fig. 2. The induced metric

$$
g_{a b}=G_{\mu \nu} \partial_{a} x^{\mu} \partial_{b} x^{\nu} \quad(a, b=\varphi, r)
$$


and its determinant are given by

$$
\begin{aligned}
g_{a b} & =\left(\begin{array}{cc}
{\left[1+z^{\prime}(r)^{2}\right] \mathrm{e}^{2 A(z)}} & 0 \\
0 & r^{2} \mathrm{e}^{2 A(z)}
\end{array}\right), \\
\operatorname{det}\left(g_{a b}\right) & =r^{2}\left[1+z^{\prime}(r)^{2}\right] \mathrm{e}^{4 A(z)},
\end{aligned}
$$

which leads to the following expression for the corresponding NG action:

$$
S_{\mathrm{NG}}=\frac{1}{\alpha^{\prime}} \int_{0}^{a} d r r \mathrm{e}^{2 A(z)} \sqrt{1+z^{\prime}(r)^{2}},
$$

where the $\varphi$ integration has been done, canceling the $2 \pi$ factor in (2.4). It is worth noting that this Lagrangian depends on the integration variable, and therefore the Hamiltonian is not a constant of motion in this case. The Euler-Lagrange equations of motion arising from this action are

$$
r \frac{z^{\prime \prime}(r)}{1+z^{\prime}(r)^{2}}+z^{\prime}(r)-2 r A^{\prime}(z)=0
$$

and the boundary conditions to be considered are

$$
z(a)=z_{1}, \quad z^{\prime}(0)=0,
$$

which correspond to a smooth surface contoured by a circular loop of radius $a$ located at the value $z_{1}$ of the coordinate $z$ orthogonal to the border. For the AdS case $A(z)=-\ln \left(\frac{z}{L}\right)$, the solution to (4.2) with the boundary conditions (4.3) is

$$
z=\sqrt{a^{2}+z_{1}^{2}-r^{2}}, \quad 0 \leq r \leq a .
$$

\section{A. Hamilton-Jacobi approach}

In this case, the NG action is a function of the radius $a$ and the location $z_{1}$ of the circular loop. The momentum canonically conjugate to $z$ and the Hamiltonian appearing in the $\mathrm{HJ}$ equation are given by

$$
\begin{aligned}
p\left(z, z^{\prime}, r\right):=\frac{\partial L\left(z, z^{\prime}, r\right)}{\partial z^{\prime}} & =\frac{1}{\alpha^{\prime}} \frac{r z^{\prime} \mathrm{e}^{2 A(z)}}{\sqrt{1+z^{\prime 2}}} \Rightarrow z^{\prime}(z, p, a) \\
& = \pm \frac{\alpha^{\prime} p}{\sqrt{r^{2} \mathrm{e}^{4 A(z)}-\alpha^{\prime 2} p^{2}}}, \\
H(z, p, r) & =p z^{\prime}(z, p, r)-L\left(z, z^{\prime}(z, p, r), r\right) \\
& =-\frac{p}{z^{\prime}(z, p, r)} \\
& =\mp \frac{1}{\alpha^{\prime}} \sqrt{r^{2} \mathrm{e}^{4 A(z)}-\alpha^{\prime 2} p^{2}} .
\end{aligned}
$$

Replacing in the HJ equation,

$$
\frac{\partial S_{\mathrm{NG}}\left(a, z_{1}\right)}{\partial a}+H\left(z_{1}, \frac{\partial S_{\mathrm{NG}}\left(a, z_{1}\right)}{\partial z_{1}}, a\right)=0
$$

leads to

$\frac{\partial S_{\mathrm{NG}}\left(a, z_{1}\right)}{\partial a} \mp \frac{1}{\alpha^{\prime}} \sqrt{a^{2} \mathrm{e}^{4 A\left(z_{1}\right)}-\alpha^{\prime 2}\left[\frac{\partial S_{\mathrm{NG}}\left(a, z_{1}\right)}{\partial z_{1}}\right]^{2}}=0$,

which implies

$$
\left[\frac{\partial S_{\mathrm{NG}}\left(a, z_{1}\right)}{\partial a}\right]^{2}+\left[\frac{\partial S_{\mathrm{NG}}\left(a, z_{1}\right)}{\partial z_{1}}\right]^{2}=\frac{1}{\alpha^{\prime 2}} a^{2} \mathrm{e}^{4 A\left(z_{1}\right)} .
$$

In the AdS case this equation is

$$
\left[\frac{\partial S_{\mathrm{NG}}\left(a, z_{1}\right)}{\partial a}\right]^{2}+\left[\frac{\partial S_{\mathrm{NG}}\left(a, z_{1}\right)}{\partial z_{1}}\right]^{2}=\frac{L^{4}}{\alpha^{\prime 2}} \frac{a^{2}}{z_{1}^{4}},
$$

whose solution with the boundary condition,

$$
\lim _{a \rightarrow 0^{+}} S_{\mathrm{NG}}\left(a, z_{1}\right) \equiv 0 \quad\left(z_{1}=\text { cte }\right),
$$

is

$$
S_{\mathrm{NG}}^{\mathrm{AdS}}\left(a, z_{1}\right)=\frac{L^{2}}{\alpha^{\prime}}\left[\sqrt{1+\frac{a^{2}}{z_{1}^{2}}}-1\right],
$$

which coincides with what is obtained by replacing the solution (4.4) in the NG action (4.1).

\section{B. Expansion in powers of the radius $a$}

An expansion of the on-shell NG action for the circular loop in powers of $a$ allows one to obtain information about the gluon condensates in the dual gauge theory [14-16]. It is not totally straightforward to perform such an expansion. This can be seen from the result (4.9) for the on-shell NG action in the AdS case. The series expansion of $S_{\mathrm{NG}}^{\mathrm{AdS}}\left(a, z_{1}\right)$ in powers of $a$ is given by

$$
\frac{\alpha^{\prime}}{L^{2}} S_{\mathrm{NG}}^{\mathrm{AdS}}\left(a, z_{1}\right)=\frac{a^{2}}{2 z_{1}^{2}}-\frac{a^{4}}{8 z_{1}^{4}}+\frac{a^{6}}{16 z_{1}^{6}}+\mathcal{O}\left(a^{7}\right),
$$

which is convergent for $\frac{a}{z_{1}}<1$. Therefore such an expansion is not suited to reproduce the behavior of $S_{\mathrm{NG}}^{\mathrm{AdS}}\left(a, z_{1}\right)$ for $z_{1} \rightarrow 0$ and $a$ fixed. Indeed, (4.9) shows that

$$
\frac{\alpha^{\prime}}{L^{2}} S_{\mathrm{NG}}^{\mathrm{AdS}}\left(a, z_{1}\right) \stackrel{z_{1} \ll 1}{=} \frac{a}{z_{1}} .
$$

In this respect it is convenient to consider the NG action in terms of the variables $w_{1}=\frac{z_{1}}{a}$ and $a$ instead of $z_{1}$ and $a$. Doing this for the AdS case gives 


$$
\frac{\alpha^{\prime}}{L^{2}} S_{\mathrm{NG}}^{\mathrm{AdS}}\left(a, w_{1} a\right)=\frac{\sqrt{1+w_{1}^{2}}}{w_{1}}-1,
$$

whose Laurent expansion for $w_{1} \ll 1$ reproduces the divergence term $1 / w_{1}$ in (4.11); this is not the case for the expansion (4.10).

Defining the action $S\left(a, w_{1}\right)$ by

$$
S\left(a, w_{1}\right)=S_{\mathrm{NG}}\left(a, w_{1} a\right)
$$

and taking into account that

$$
\begin{aligned}
& \frac{\partial S_{\mathrm{NG}}\left(a, z_{1}\right)}{\partial a}=\frac{\partial S\left(a, w_{1}\right)}{\partial a}-\frac{w_{1}}{a} \frac{\partial S\left(a, w_{1}\right)}{\partial w_{1}}, \\
& \frac{\partial S_{\mathrm{NG}}\left(a, z_{1}\right)}{\partial z_{1}}=\frac{1}{a} \frac{\partial S\left(a, w_{1}\right)}{\partial w_{1}},
\end{aligned}
$$

the $\mathrm{HJ}$ equation is rewritten as follows:

$$
\begin{gathered}
a^{2}\left[\frac{\partial S\left(a, w_{1}\right)}{\partial a}\right]^{2}+\left(1+w_{1}^{2}\right)\left[\frac{\partial S\left(a, w_{1}\right)}{\partial w_{1}}\right]^{2} \\
-2 w_{1} a \frac{\partial S\left(a, w_{1}\right)}{\partial a} \frac{\partial S\left(a, w_{1}\right)}{\partial w_{1}} \\
=\frac{1}{\alpha^{2}} a^{4} \mathrm{e}^{4 A\left(w_{1} \cdot a\right)},
\end{gathered}
$$

and the boundary condition (4.8) is now

$0=\lim _{a \rightarrow 0^{+}} S_{\mathrm{NG}}\left(a, z_{1}\right)=\lim _{a \rightarrow 0^{+}} S\left(a, \frac{z_{1}}{a}\right) \quad\left(z_{1}=\mathrm{cst}.\right)$.

Next the following power series expansion is considered:

$$
S\left(a, w_{1}\right)=\frac{L^{2}}{\alpha^{\prime}} \sum_{n=0}^{\infty} s_{2 n}\left(w_{1}\right) a^{2 n},
$$

and replacing this expansion in (4.12) leads to

$$
\begin{aligned}
& \sum_{n=0}^{\infty}\left(\sum_{k=0}^{n} 4(k+1)(n-k+1) s_{2(k+1)}\left(w_{1}\right) s_{2(n-k+1)}\left(w_{1}\right)\right) a^{2 n+4} \\
& -2 w_{1} \sum_{n=0}^{\infty}\left(\sum_{k=0}^{n} 2(k+1) s_{2(k+1)}\left(w_{1}\right) s_{2(n-k)}^{\prime}\left(w_{1}\right)\right) a^{2 n+2} \\
& +\left(1+w_{1}^{2}\right) \sum_{n=0}^{\infty}\left(\sum_{k=0}^{n} s_{2 k}^{\prime}\left(w_{1}\right) s_{2(n-k)}^{\prime}\left(w_{1}\right)\right) a^{2 n} \\
& -\sum_{n=0}^{\infty} \beta_{2 n} w_{1}^{2 n-4} a^{2 n}=0
\end{aligned}
$$

where $\beta_{m}$ are the power series expansion coefficients of $\mathrm{e}^{4 A\left(w_{1} a\right)}$, i.e., $\mathrm{e}^{4 A\left(w_{1} a\right)}=\frac{L^{4}}{\left(w_{1} a\right)^{4}} \mathrm{e}^{4 f\left(w_{1} a\right)}=\frac{L^{4}}{\left(w_{1} a\right)^{4}} \sum_{n=0}^{\infty} \beta_{2 n}\left(w_{1} a\right)^{2 n}$.

These coefficients can be written as polynomials in the $\alpha$ coefficients appearing in (2.3). Equating to zero the coefficient of $a^{n}$ in the left-hand side of (4.15) leads to

$$
\begin{aligned}
& 2\left(1+w_{1}^{2}\right) s_{0}^{\prime}\left(w_{1}\right) s_{2 n}^{\prime}\left(w_{1}\right)-4 n w_{1} s_{0}^{\prime}\left(w_{1}\right) s_{2 n}\left(w_{1}\right) \\
& \quad+\sum_{k=1}^{n-1}\left\{\left(1+w_{1}^{2}\right) s_{2 k}^{\prime}\left(w_{1}\right) s_{2(n-k)}^{\prime}\left(w_{1}\right)\right. \\
& \quad+4 k(n-k) s_{2 k}\left(w_{1}\right) s_{2(n-k)}\left(w_{1}\right) \\
& \left.\quad-4 w_{1} k s_{2 k}\left(w_{1}\right) s_{2(n-k)}^{\prime}\left(w_{1}\right)\right\}-\beta_{2 n} w_{1}^{2 n-4}=0,
\end{aligned}
$$

valid for $n=0,1,2, \ldots$. The boundary condition (4.13) leads to

$$
\begin{aligned}
\lim _{a \rightarrow 0^{+}} S\left(a, z_{1} / a\right) & =0 \Leftrightarrow \lim _{a \rightarrow 0^{+}} s_{2 n}\left(z_{1} / a\right) \cdot a^{2 n}=0 \\
\forall n, z_{1} & =\text { cst. }
\end{aligned}
$$

For a given $n$, Eq. (4.17) involves the functions $s_{2 k}\left(w_{1}\right)$ and $s_{2 k}^{\prime}\left(w_{1}\right)$ for $0 \leq k \leq n$. Therefore starting with $n=0$, the resulting equation only involves $s_{0}\left(w_{1}\right)$ and $s_{0}^{\prime}\left(w_{1}\right)$, and when solving for them they can be replaced in the equation for $n=1$ to get $s_{2}\left(w_{1}\right)$ and $s_{2}^{\prime}\left(w_{1}\right)$ and so on. The equation for $n=0$ and its solution satisfying the boundary condition (4.18) are

$$
\left[s_{0}^{\prime}\left(w_{1}\right)\right]^{2}=\frac{1}{w_{1}^{4}\left(1+w_{1}^{2}\right)} \Rightarrow s_{0}\left(w_{1}\right)=+\left(\frac{\sqrt{1+w_{1}^{2}}}{w_{1}}-1\right),
$$

where the sign in the second equation has been chosen so as to get a positive area for a nonvanishing radius. For the cases with $n=1,2, \ldots$, the differential equations to be considered are of the form

$A^{(2 n)}\left(w_{1}\right) s_{2 n}^{\prime}\left(w_{1}\right)+B^{(2 n)}\left(w_{1}\right) s_{2 n}\left(w_{1}\right)+C^{(2 n)}\left(w_{1}\right)=0$,

and the general solution to this equation is

$$
\begin{aligned}
s_{2 n}\left(w_{1}\right) & =c^{(2 n)} \mathrm{e}^{F\left(w_{1}\right)}-\mathrm{e}^{F\left(w_{1}\right)} \int_{0}^{w_{1}} \mathrm{e}^{-F(x)} \frac{C^{(2 n)}(x)}{A^{(2 n)}(x)} \mathrm{d} x, \\
F\left(w_{1}\right) & =-\int_{0}^{w_{1}} \frac{B^{(2 n)}(x)}{A^{(2 n)}(x)} \mathrm{d} x,
\end{aligned}
$$

where $c^{(2 n)}$ is a constant to be determined using the boundary condition (4.18). Equation (4.17) implies that 


$$
\frac{B^{(2 n)}(x)}{A^{(2 n)}(x)}=-2 n \frac{w_{1}}{1+w_{1}^{2}} \Rightarrow F\left(w_{1}\right)=\ln \left[\left(1+w_{1}^{2}\right)^{n}\right],
$$

and replacing in (4.21) leads to

$$
\begin{aligned}
s_{2 n}\left(w_{1}\right)= & c^{(2 n)}\left(1+w_{1}^{2}\right)^{n}+\frac{1}{2}\left(1+w_{1}^{2}\right)^{n} \\
& \times \int_{0}^{w_{1}} \frac{x^{2}}{\left(1+x^{2}\right)^{n+\frac{1}{2}}} C^{(2 n)}(x) \mathrm{d} x,
\end{aligned}
$$

where the following equalities were employed: $A^{(2 n)}(x)=$ $2\left(1+x^{2}\right) s_{0}^{\prime}(x)=-\frac{2 \sqrt{1+x^{2}}}{x^{2}}$. Imposing the boundary condition (4.18) leads to

$$
c^{(2 n)}=-\frac{1}{2} \int_{0}^{+\infty} \frac{x^{2}}{\left(1+x^{2}\right)^{n+\frac{1}{2}}} C^{(2 n)}(x) \mathrm{d} x,
$$

which replacing in (4.22) gives

$s_{2 n}\left(w_{1}\right)=-\frac{1}{2}\left(1+w_{1}^{2}\right) \int_{w_{1}}^{+\infty} \frac{x^{2}}{\left(1+x^{2}\right)^{n+\frac{1}{2}}} C^{(2 n)}(x) \mathrm{d} x$.

The functions $C^{(2 n)}$ appearing in this expression are obtained from (4.17),

$$
\begin{aligned}
C^{(2 n)}(x)= & \sum_{k=1}^{n-1}\left\{\left(1+x^{2}\right) s_{2 k}^{\prime}(x) s_{2(n-k)}^{\prime}(x)\right. \\
& +4 k(n-k) s_{2 k}(x) s_{2(n-k)}(x) \\
& \left.-4 w_{1} k s_{2 k}(x) s_{2(n-k)}^{\prime}(x)\right\}-\beta_{2 n} x^{2 n-4},
\end{aligned}
$$

and for $n=0,1,2,3$ the results for $C^{(2 n)}(x)$ and $s_{2 n}\left(w_{1}\right)$ are given in Appendix A.

In addition, the special cases where only one of the $\beta_{2 n}$ is nonvanishing ${ }^{3}$ can be exactly calculated for any $n$. In such cases,

$$
C^{(2 n)}(x)=-\beta_{2 n} x^{2 n-4}
$$

which leads to

$$
\begin{aligned}
s_{2 n} & =-\frac{1}{4}\left(1+w_{1}^{2}\right) \int_{w 1}^{\infty} d x \frac{x^{2}}{\left(1+x^{2}\right)^{n+\frac{1}{2}}} C^{(2 n)}(x) \\
& =\frac{\beta_{2 n}}{2}\left(1+w_{1}^{2}\right) \int_{w 1}^{\infty} d x \frac{x^{2 n-2}}{\left(1+x^{2}\right)^{n+\frac{1}{2}}} \\
& =\frac{\beta_{2 n}}{2}\left(1+w_{1}^{2}\right)\left(\frac{1-w_{1}^{2 n-1}\left(1+w_{1}^{2}\right)^{\frac{1}{2}-n}}{2 n-1}\right) \\
& \stackrel{w_{1} \rightarrow 0}{=} \frac{\beta_{2 n}}{2(2 n-1)} .
\end{aligned}
$$

Switching on other $\beta$ 's will give additional contributions to the $s_{2 n}$. However, the numerical coefficient in front of $\beta_{2 n}$ will be the same as above.

\section{SUBSTRACTION}

In the previous section the NG action has been regularized by locating the border of the surface at a nonzero value $z_{1}$ of the coordinate $z$. Other regularizations can also be employed, and one example of these will be considered in Sec. VI. Once regularized the next step is to define a substraction. The important properties of a substraction are that it should remove the divergence terms and that the substracted quantity should not depend on the regularization employed. This is normally achieved by means of substracting to the regularized quantity a certain number of terms of its expansion around certain values of the parameters on which it depends. In the present case, the calculation of the NG action, the parameters characterizing the calculation are purely geometrical and consist in the ones that describe the contour of the loop at the border of the surface whose area is computed. In both cases considered in this work the divergences are proportional to the perimeter of the loop at the border of the surface whose area is computed. This last fact can be derived for the rectangular loop by direct computation as (3.9) shows. ${ }^{4}$ For the circular loop this can be derived as in Sec. III. A in [16]. This motivates the following definitions of the substracted NG action for the rectangular and circular loops:

$$
\begin{array}{cc}
\text { Rectangular } & S_{\mathrm{NG}}^{R \text {,subs. }}=\lim _{\Lambda \rightarrow \infty}\left(S_{\mathrm{NG}}-\left.S_{\mathrm{NG}}\right|_{R=R_{0}}\right) \\
\text { Circular } \quad S_{\mathrm{NG}}^{C, \text { subs. }}=\lim _{\Lambda \rightarrow \infty}\left(S_{\mathrm{NG}}-\left.S_{\mathrm{NG}}\right|_{a=a_{0}}-\left.\frac{\partial S_{\mathrm{NG}}}{\partial a}\right|_{a=a_{0}}\left(a-a_{0}\right)\right),
\end{array}
$$

where $R$ denotes the interquark separation, $a$ denotes the radius of the circular loop, and $R_{0}, a_{0}$ denote the corresponding substraction points. In the above expressions $\lim _{\Lambda \rightarrow \infty}$ indicates that the cutoff limit should be taken.

\footnotetext{
${ }^{3}$ It is remarked that the exact solvability of these cases is not obvious from the point of view of solving the corresponding equations of motion (4.2).

${ }^{4}$ For the rectangular loop, in the limit $T \rightarrow \infty$, the perimeter is, up to a small correction, the same as $2 T$.
} 


\section{A. The substracted rectangular loop}

For the rectangular loop the above definition leads to the following expression for the substracted NG action:

$$
\begin{aligned}
S_{\mathrm{NG}}^{R \text {,subs. }}= & \lim _{z_{1} \rightarrow 0} \frac{T}{2 \pi \alpha^{\prime}}\left(R e^{2 A\left(z_{0}(R)\right)}-R_{0} e^{2 A\left(z_{0}\left(R_{0}\right)\right)}\right. \\
& +2 \int_{z_{1}}^{z_{0}(R)} \sqrt{\mathrm{e}^{4 A(z)}-\mathrm{e}^{4 A\left(z_{0}(R)\right)}} d z \\
& \left.-2 \int_{z_{1}}^{z_{0}\left(R_{0}\right)} \sqrt{\mathrm{e}^{4 A(z)}-\mathrm{e}^{4 A\left(z_{0}\left(R_{0}\right)\right)}} d z\right),
\end{aligned}
$$

and this expression is finite. This can be seen by noting that the integrands of the two last terms have the same singularity for $z \rightarrow 0$, with the same coefficient. Therefore they cancel leading to a finite expression.

For the case of confining wrap factors, i.e., when $e^{2 A(z)}$ has a minimum at a finite value of $z$, it leads to a linear potential between static quarks, with a string tension given by ${ }^{5}$ $e^{2 A\left(z_{0}(R)\right)}$ that does not depend on the substraction point $R_{0}$. In addition, there are constant terms that do depend on the substraction point. This can be seen explicitly using (3.9).

\section{B. The substracted circular loop}

The result for the regularized NG action is

$$
S_{\mathrm{NG}}\left(a, w_{1}\right)=\frac{L^{2}}{\alpha^{\prime}}\left(\left(\frac{\sqrt{1+w_{1}^{2}}}{w_{1}}-1\right)+\sum_{n=1}^{\infty} s_{2 n}\left(w_{1}\right) a^{2 n}\right),
$$

where the coefficients $s_{2 n}\left(w_{1}\right)$ are given in Appendix A. Replacing the relation $w_{1}=\frac{z_{1}}{a}$ in this last relation leads to

$$
S_{\mathrm{NG}}\left(a, z_{1}\right)=\frac{L^{2}}{\alpha^{\prime}}\left(\sqrt{1+\frac{a^{2}}{z_{1}^{2}}}-1+\sum_{n=1}^{\infty} s_{2 n}\left(\frac{z_{1}}{a}\right) a^{2 n}\right)
$$

and replacing in (5.1) leads to

$$
\begin{aligned}
S_{\mathrm{NG}}^{C, \text { subs. }} & =\lim _{z_{1} \rightarrow 0} \frac{L^{2}}{\alpha^{\prime}}\left(\sqrt{1+\frac{a^{2}}{z_{1}^{2}}}-1-\sqrt{1+\frac{a_{0}^{2}}{z_{1}^{2}}}+1-\frac{\left(a-a_{0}\right)}{z 1} \frac{1}{\sqrt{1-\frac{z_{1}^{2}}{a^{2}}}}\right)+\sum_{n=1}^{\infty}\left(s_{2 n}\left(\frac{z_{1}}{a}\right) a^{2 n}-s_{2 n}\left(\frac{z_{1}}{a_{0}}\right) a_{0}^{2 n}\right) \\
& =\frac{L^{2}}{\alpha^{\prime}}\left(-\delta_{a_{0}, 0}+\sum_{n=1}^{\infty}\left(s_{2 n}(0) a^{2 n}-s_{2 n}(0) a_{0}^{2 n}\right)\right) .
\end{aligned}
$$

This expression shows that the substracted action is finite and that only the constant term independent of $a$ depends on the substraction point $a_{0}$.

It is remarked that for the circular loop the substracted action presented in this work coincides with the one given in [16] up to terms proportional to the perimeter. The important difference between both is that the last procedure is given in terms of the particular regularization chosen in that work. This is not the case for the one given here since it does not depend on the regularization employed.

\section{COMPARISON WITH OTHER COMPUTATIONS}

In this section the computations done above are compared with the analogous ones computed using the $\epsilon$-scheme. This is particularly relevant since, although the results coincide for the case of the rectangular loop, this is not the case for the circular loop. The result for the expansion coefficients $s_{2 n}$ of the substracted NG action in powers of the radius $a$ for the HJ scheme and ${ }^{6} \tilde{a}$ for the $\epsilon$-scheme for the case of the circular loop do not coincide

\footnotetext{
${ }^{5}$ For these wrap factors the minimum of the potential coincides with $z_{0}$, the maximum value of $z$ attained by the surface. In other words, the minimal surface sticks to the minimum of $e^{2 A(z)}$ in order to minimize its area.

${ }^{6} \tilde{a}$ denotes the radius of the contour at the border $z=0$.
}

between both schemes in the limit $z_{1} \rightarrow 0$. he source of this discrepancy is analyzed below. In order to do this it is necessary to consider the process of regularization/substraction involved in the $\epsilon$-scheme. The $\epsilon$-regularization scheme is depicted in Fig. 3.

For the nonconformal case this scheme is considered in [15]. It consists in considering the solution of the minimizing equations (4.2) for a surface whose boundary is located at $z=0$. The area of such a surface is divergent. This is regularized by computing another area that corresponds to integrating the same integrand up to a value of the border coordinate orthogonal to this boundary an amount $\epsilon$ less ${ }^{7}$ than the one of the boundary located at $z=0$. This area is grayed in Fig. 3. This approach can be alternatively obtained by considering the solution to the equation of motion (4.2) with the boundary conditions (4.3) and taking the limit $z_{1} \rightarrow 0$ in the integrand of the NG action. This last approach was employed in [16]. In the subsections appearing below the two schemes are analyzed in detail for both the rectangular and the circular loops.

\footnotetext{
${ }^{7}$ In Ref. [15], for the case of the circular loop of radius $\tilde{a}$ at $z=0$, the $\epsilon$ employed in this work is written as $\epsilon=$ $\tilde{a}-\sqrt{\tilde{a}^{2}-z_{1}^{2}} \stackrel{z_{1} \rightarrow 0}{=} \frac{z_{1}^{2}}{2 \tilde{a}}$. This relation between $\epsilon$ and $z_{1}$, is the one that would correspond if the minimal surface would be the AdS one. The $z_{1}$ in this work is called $\epsilon$ in Ref. [15].
} 


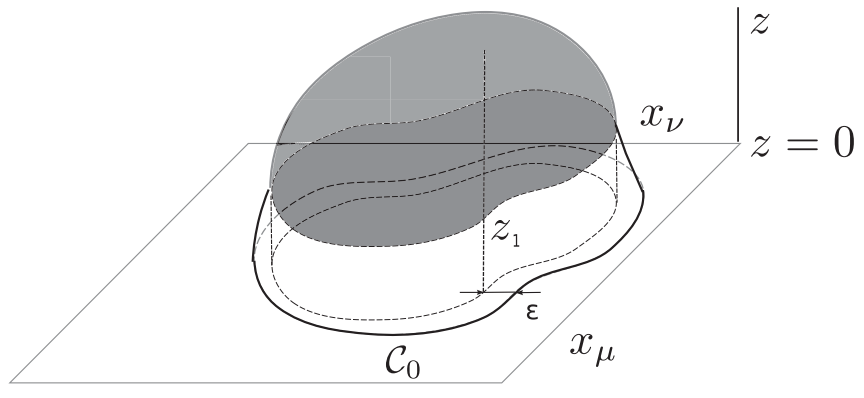

FIG. 3. The $\epsilon$ regularization.

\section{A. The rectangular loop}

The solution to the equations of motion for this case can be obtained by noting that the Hamiltonian corresponding to the Lagrangian appearing in (3.1) is a constant of motion $E$, given by

$$
E=-\frac{e^{2 A}}{\sqrt{1+z^{\prime}(x)^{2}}},
$$

where the ' indicates the derivative with respect to $x$. This leads to the following linear ordinary differential equation:

$$
z^{\prime}(x)=\sqrt{\frac{e^{4 A}}{E^{2}}-1}
$$

from which it is simple to obtain $x$ as a function of $z$,

$$
x(z)=a-\int_{z_{1}}^{z} d z \frac{E}{\sqrt{e^{4 A}-E^{2}}} .
$$

This solution satisfies the boundary condition $x\left(z_{1}\right)=a$, and therefore corresponds to a pair of static quarks located at $z=z_{1}$ and separated a distance $2 a$. The relation between $z_{1}, z_{0}, E$, and $a$ can be obtained using that by definition $x\left(z_{0}\right)=0$, leading to

$$
a=\int_{z_{1}}^{z_{0}} d z \frac{E}{\sqrt{e^{4 A}-E^{2}}} .
$$

In order to insert this solution in the Nambu-Goto action it is convenient to consider an alternative embedding of the same surface in the five-dimensional space. In this embedding one considers $x$ as a function of $z$. It is given by

$$
\begin{aligned}
x^{1} & =t, \quad t \in\left[-\frac{T}{2}, \frac{T}{2}\right], \\
x^{i} & =x=x(z), \quad x \in[-a, a], \\
x^{k} & =0, \quad \forall k \neq i, \\
x^{5} & =z .
\end{aligned}
$$

The NG action is given in terms of this embedding by

$$
S_{\mathrm{NG}}=\frac{1}{2 \pi \alpha^{\prime}} \int_{-\frac{T}{2}}^{\frac{T}{2}} d t \int_{z_{1}}^{z_{0}} d z \mathrm{e}^{2 A(z)} \sqrt{1+x^{\prime}(z)^{2}},
$$

where now the ' indicates the derivative with respect to $z, z_{1}$ is the location of the loop contour, and $z_{0}$ is the maximum value of $z$ attained by the minimal surface. Evaluation of the NG action in the solution of the equations of motion amounts to replace (6.1) in (6.3). The integrand in (6.3) is independent of $z_{1}$, because $x^{\prime}(z)$ is independent of $z_{1}$ as implied by (6.1). The lower integration limit is common to the $\mathrm{HJ}$ and the $\epsilon$-regularization. The upper limit of integration is to be determined as a function of $a$ and $z_{1}$ by means of (6.2). For the $\epsilon$-regularization this amounts to take $z_{1} \rightarrow 0$ in (6.2). The function $z_{0}\left(a, z_{1}\right)$ just gives the maximum of the minimal surface, and this function has no singularities for any value of $z_{1} \geq 0$. Therefore taking the limit $z_{1} \rightarrow 0$ before or after the integration makes no difference. Thus the result for the substracted NG action is the same for both schemes.

\section{B. Circular loop}

The results for the expansion coefficients $s_{2 n}$ of the NG action in powers of the radius $a$ for the case of the circular loop are shown in Table I. The first column corresponds to the results computed with the HJ scheme. The second column corresponds to the results computed in [16] using the $\epsilon$-scheme and taking the radius of the contour at the border as the expansion parameter. The first two rows in column $\epsilon$ also coincide with the results in [15], which takes $\alpha_{n}=\delta_{n 2} \alpha_{2}$. In the $\epsilon$-computation the loop is located at $z=0$. These two approaches were considered in [17] for the case of the supersymmetric conformal theory. There it is shown that in the AdS case for smooth surfaces both regularizations lead to the same results, except with respect to zigzag symmetry. The HJ regularization respects this symmetry but the $\epsilon$-regularization does not.

TABLE I. Results for the expansion coefficients of the NG action for the circular loop in powers of the radius $a$. The results for $s_{6}, s_{8}$, and $s_{10}$ are given taking $\alpha_{2}=0$.

\begin{tabular}{lcc}
\hline \hline$s_{2 n}$ & $\mathrm{HJ}$ & $\epsilon$ \\
\hline$s_{2}$ & $2 \alpha_{2}$ & $\frac{10}{3} \alpha_{2}$ \\
$s_{4}$ & $\frac{2}{3}(17-24 \log 2) \alpha_{2}^{2}+\frac{2}{3} \alpha_{4}$ & $\frac{14}{9}(17-24 \log 2) \alpha_{2}^{2}+\frac{14}{9} \alpha_{4}$ \\
$s_{6}$ & $\frac{2}{5} \alpha_{6}$ & $\frac{3}{5} \alpha_{6}$ \\
$s_{8}$ & $\frac{2}{945}(2111-3360 \log 2) \alpha_{4}^{2}+\frac{2}{7} \alpha_{8}$ & $\frac{11}{5670}\left[(2111-3360 \log 2) \alpha_{4}^{2}+270 \alpha_{8}\right]$ \\
$s_{10}$ & $\frac{4}{1575}(2999-5040 \log 2) \alpha_{4} \alpha_{6}+\frac{2}{9} \alpha_{10}$ & $\frac{13}{4725}\left[(2999-5040 \log 2) \alpha_{4} \alpha_{6}+175 \alpha_{10}\right]$ \\
\hline \hline
\end{tabular}


Below it is shown that for a particular choice of $\beta_{n}$ coefficients, which can be exactly solved, the $\epsilon$-regularization leads to a wrong result if the radius $\tilde{a}$ corresponding to the intersection of the minimal surface with the plane $z=0$ is employed as the expansion parameter. For the case of the HJ regularization the radius $a$ of the border of the grayed area in Fig. 3 is the expansion parameter. In this respect it is worth noting that the radius $a$ is intrinsic to the geometrical problem to be considered. This is so because $a$ is the radius of the border of the minimal surface whose area is computed. In contrast, $\tilde{a}$ is a quantity that is related to the way one chooses to regularize in the $\epsilon$-scheme the calculation of this area. ${ }^{8}$ It is also important to recall that the substraction considered in Sec. V is given in terms of the radius of the border of the minimal surface whose area is to be computed, and this radius is $a$ and not $\tilde{a}$. Indeed, at that level, $\tilde{a}$ is not even defined since the substraction should not depend on the regularization employed.

In addition, it is also shown that if instead of taking the limit $z_{1} \rightarrow 0$ in the integrand (which corresponds to the $\epsilon$-regularization), terms up to order $\mathcal{O}\left(z_{1}^{3}\right)$ are kept, then the results also coincide with the HJ approach.

\section{For solvable case e-scheme gives wrong result}

In the following it is shown that for a particular choice of the $\beta$-coefficients the NG action for the circular loop can be calculated without approximations. This choice corresponds to taking

$$
\beta_{n}=\beta_{2} \delta_{n 2} .
$$

The above assertion can readily be understood since this case corresponds to a warp factor that leads to a flat metric; indeed in such a case,

$$
e^{2 A(z)}=\frac{1}{z^{2}} \beta_{2} z^{2}=\beta_{2} \Rightarrow A^{\prime}(z)=0,
$$

the solution of the equation of motion (4.2) in this case is

$$
z^{\prime}(r)=0
$$

Therefore the on-shell NG action has no divergences and is given by

$$
\begin{aligned}
S_{\mathrm{NG}} & =\frac{1}{\alpha^{\prime}} \int_{0}^{a} d r \mathrm{e}^{2 A(z)} \sqrt{1+z^{\prime}(r)^{2}} \\
& =\frac{1}{\alpha^{\prime}} \int_{0}^{a} d r r \mathrm{e}^{2 A(z)}=\frac{1}{\alpha^{\prime}} \int_{0}^{a} d r r \beta_{2} \\
& =\frac{1}{\alpha^{\prime}} \frac{\beta_{2}}{2} a^{2}=\frac{1}{\alpha^{\prime}} 2 \alpha_{2} a^{2} .
\end{aligned}
$$

This result coincides with the one obtained using the HJ scheme and not with the one corresponding to the

\footnotetext{
${ }^{8}$ In mathematical terms, $\tilde{a}$ is a quantity dependent on the way the loop contour is embedded in five-dimensional space, and therefore is not intrinsic to the geometry of the surface under consideration.
}

$\epsilon$-scheme. Thus showing that at least in this case the $\epsilon$-scheme, using the radius $\tilde{a}$ as the expansion parameter, gives a wrong answer.

\section{Using a as expansion parameter in the 6 -scheme gives the same results as the $\mathrm{HJ}$ scheme}

Both regularizations are directly related to one another. Indeed, both are solutions of the same minimizing equations with different boundary conditions. It is therefore geometrically clear that there should exist a value of $\epsilon$ and a location of the contour at $z=0$, such that a line parallel to the $z$ axis should intersect the minimization surface at $z=z_{1}$. This is shown in Fig. 3. If the radius of the circular loop located at $z=0$ in the $\epsilon$-regularization is denoted by $\tilde{a}$, which implies that

$$
z(\tilde{a})=0,
$$

then the radius $a$ is defined as the one that corresponds to the circle that intersects the minimal surface at $z=z_{1}$, i.e.,

$$
z(a)=z_{1} \text {. }
$$

The discrepancy between both computations appears when the expansion parameter in the $\epsilon$-scheme is taken to be $\tilde{a}$. Although in the cutoff limit $\left(\epsilon \rightarrow 0, z_{1} \rightarrow 0\right)$ both values coincide $\left(\lim _{\epsilon \rightarrow 0} \tilde{a}=a\right.$ ), it is not the same to take the limit before or after doing the integration. This is so because the integration limit depends on $\epsilon$ or equivalently on $z_{1}$. The important point is that if one replaces $\tilde{a}$ in terms of $a$ and $z_{1}$, in the expansion of the integrand of the NG action in powers of $\tilde{a}$, then upon integration the HJ result for the coefficients of the expansion of the NG action in powers of $a$ are obtained.

The relation between $\tilde{a}$ and $a$ can be obtained in the following way. First it is noted that for the case of interest $z_{1} \ll a$, and therefore it is adequate to express $\tilde{a}$ as a series in powers of $z_{1}$. The coefficients of this series can be obtained by taking the solution of the equations of motion (4.2) with the boundary condition (6.4) and solving Eq. (6.5). This leads to the following expression of $\tilde{a}$ in terms of $a$ :

$$
\tilde{a}=a+\frac{z_{1}^{2}}{2 a}+\frac{4}{3} \alpha_{2} z_{1}^{3}+\mathcal{O}\left(z_{1}^{4}\right) .
$$

\section{Expanding the integrand up to $\mathcal{O}\left(z_{1}^{3}\right)$ gives the same results as the $\mathrm{HJ}$ scheme}

The results for the $\mathrm{HJ}$ regularization can be computed either by the HJ approach or by solving the differential equation and replacing the solution in the NG action. Both methods lead to the same results. In the second approach there appear terms in the integrand that go to zero when $z_{1} \rightarrow 0$ but survive after integration. These terms are responsible for the discrepancy with the $\epsilon$-regularization which, as mentioned before, is equivalent to taking the limit $z_{1} \rightarrow 0$ in the integrand before performing the integral. In Appendix B a concrete example is considered which shows how these terms arise for the case of the coefficient $s_{2}$. 


\section{CONCLUDING REMARKS}

In this work the $\mathrm{HJ}$ approach has been employed for the calculation of minimal areas on asymptotically AdS spaces. These calculations are relevant, from the holographic point of view, in obtaining expectation values of Wilson loops in the gauge theory living at the border of these spaces. These expectation values are related to the phenomenologically relevant condensates of the gauge theory [16]. In this respect it is worth noting the following:

(i) This approach directly calculates the minimal area without need to solve the equations of motion and replace the solution in the NG action. This makes the calculation more direct and in practice much simpler. In this respect it is noteworthy that for the cases considered at the end of Sec. IV B, the expansion coefficients of $S_{\mathrm{NG}}$ can be exactly computed.
This calculation is not obvious from the point of view of solving the equations of motion.

(ii) In this approach variations of the on-shell classical action under changes in its boundary conditions are studied. The location of the loop contour is one of these conditions. Therefore the HJ-approach also leads to a natural regularization, which consists in moving the location of the contour out of the border.

(iii) Regarding the issue of regularization schemes it was shown that different schemes lead to different results. For the AdS case if one requires zigzag symmetry to be respected then, as shown in [17], the HJ scheme should be chosen. In addition, it is shown that for certain solvable non-AdS cases the $\epsilon$-regularization leads to wrong results if the radius $\tilde{a}$ of the contour at the border is employed as the expansion parameter. That parameter is regularization dependent and geometrically nonintrinsic.

\section{APPENDIX A: THE FIRST TERMS IN THE EXPANSION IN POWERS OF THE RADIUS}

$$
\begin{aligned}
& C^{(2)}(x)=-\frac{\beta_{2}}{x^{2}}, \\
& s_{2}\left(w_{1}\right)=-w_{1} \sqrt{1+w_{1}^{2}} \frac{\beta_{2}}{2}+\frac{\beta_{2}}{2}\left(1+w_{1}^{2}\right) \stackrel{w_{1} \rightarrow 0}{=} \frac{\beta_{2}}{2}=2 \alpha_{2}, \\
& C^{(4)}(x)=\left(1+x^{2}\right) s_{2}^{\prime}(x)^{2}-4 x s_{2}^{\prime}(x) s_{2}(x)+4 s_{2}(x)^{2}-\beta_{4}, \\
& s_{4}\left(w_{1}\right)=\frac{1}{24}\left\{4 \beta_{4}+4 \beta_{4}\left(2+w_{1}^{2}\right) w_{1}^{2}-4 \beta_{4} w_{1}^{3} \sqrt{w_{1}^{2}+1}+3 \beta_{2}^{2}\left[w_{1}^{2}\left(9 w_{1}^{2}-9 w_{1} \sqrt{w_{1}^{2}+1}+14\right)-8 \sqrt{w_{1}^{2}+1}+5 w_{1}\right]\right. \\
& \left.+12 \beta_{2}^{2}\left(1+w_{1}^{2}\right)^{2}\left[2 \arcsin \left(w_{1}\right)-\log \left(1+w_{1}^{2}\right)-\log (4)\right]\right\} \\
& \stackrel{w_{1} \rightarrow 0}{=} \frac{\beta_{4}}{6}+\beta_{2}^{2}\left(\frac{5}{8}-\log (2)\right)=\frac{2}{3} \alpha_{4}+\frac{1}{3} \alpha_{2}^{2}(34-24 \log (4)), \\
& C^{(6)}(x)=2\left(1+x^{2}\right) s_{2}^{\prime}(x) s_{4}^{\prime}(x)-8 x s_{4}(x) s_{2}^{\prime}(x)-4 x s_{2}(x) s_{4}^{\prime}(x)+16 s_{2}(x) s_{4}(x)-\beta_{6} x^{2}, \\
& s_{6}\left(w_{1}\right)=-\frac{1}{48}\left(1+w_{1}^{2}\right)^{3}\left\{-\frac{24}{5} \beta_{6}-24 \beta_{2}^{3} \log ^{2}(4)+3 \beta_{2}^{3}[36 \log (4)-73]+\frac{4}{3} \beta_{2} \beta_{4}[24 \log (4)-59]\right. \\
& -\frac{8\left(3 \beta_{2}^{2}+\beta_{4}\right) \beta_{2}}{\left(1+w_{1}^{2}\right)^{2}}+\frac{204 \beta_{2}^{3}+48 \beta_{2} \beta_{4}}{1+w_{1}^{2}}-24 \beta_{2}^{3} \log ^{2}\left(1+w_{1}^{2}\right)-96 \beta_{2}^{3} \log (2) \log \left(1+w_{1}^{2}\right) \\
& +4\left(27 \beta_{2}^{3}+8 \beta_{2} \beta_{4}\right) \log \left(1+w_{1}^{2}\right)+\frac{192 \beta_{2}^{3} w_{1} \arcsin \left(w_{1}\right)}{\sqrt{1+w_{1}^{2}}}+\frac{96 \beta_{2}^{3} \arcsin \left(w_{1}\right)}{1+w_{1}^{2}} \\
& -\frac{48 \beta_{2}^{3} \log \left(4+4 w_{1}^{2}\right)\left(1+2 w_{1} \sqrt{1+w_{1}^{2}}-2\left(1+w_{1}^{2}\right) \arcsin \left(w_{1}\right)\right)}{w_{1}^{2}+1} \\
& -96 \beta_{2}^{3} \arcsin ^{2}\left(w_{1}\right)-216 \beta_{2}^{3} \arcsin \left(w_{1}\right)-64 \beta_{2} \beta_{4} \arcsin \left(w_{1}\right) \\
& \left.+w_{1} \frac{72 \beta_{6} w_{1}^{4}+45 \beta_{2}^{3}\left(73 w_{1}^{4}+112 w_{1}^{2}+40\right)+20 \beta_{2} \beta_{4}\left(59 w_{1}^{4}+104 w_{1}^{2}+48\right)}{15\left(1+w_{1}^{2}\right)^{5 / 2}}\right\} \\
& \stackrel{w_{1} \rightarrow 0}{=} \frac{\beta_{6}}{10}+\frac{1}{48} \beta_{2}^{3}(39+8 \log (2)(\log (4096)-15))+\frac{1}{36} \beta_{4} \beta_{2}(29-48 \log (2)) \\
& =\frac{2}{5} \alpha_{6}+\frac{1}{45}\left(4 \alpha_{2}^{3}(887+120 \log (2)(\log (4096)-19))+4 \alpha_{4} \alpha_{2}(163-240 \log (2))\right) \text {. }
\end{aligned}
$$


In addition, assuming $\beta_{2}=0$, it is possible to compute up to $s_{10}$, and the results are

$$
\begin{aligned}
& s_{8}\left(w_{1}\right)=\left(w_{1}^{2}+1\right)^{4}\left(\frac{\beta_{8}}{14}+\beta_{4}^{2}\left(\frac{623}{1080}-\frac{4 \log (2)}{9}\right)\right) \\
& +\frac{1}{7560} \beta_{4}^{2}\left(-2520-8400 w_{1}^{2}-9240 w_{1}^{4}-3360 w_{1}^{6}-3360 w_{1} \sqrt{w_{1}^{2}+1}\right. \\
& -10640 w_{1}^{3} \sqrt{w_{1}^{2}+1}-11536 w_{1}^{5} \sqrt{w_{1}^{2}+1}-4361 w_{1}^{7} \sqrt{w_{1}^{2}+1} \\
& \left.+-1680\left(w_{1}^{2}+1\right)^{4} \log \left(w_{1}^{2}+1\right)+3360\left(w_{1}^{2}+1\right)^{4} \sinh ^{-1}\left(w_{1}\right)\right)-\frac{540}{7560} \beta_{8} \sqrt{w_{1}^{2}+1} w_{1}^{7} \\
& \stackrel{w_{1} \rightarrow 0}{=} \frac{\beta_{8}}{14}+\beta_{4}^{2} \frac{(1841-3360 \log (2))}{7560}=\frac{2 \alpha_{8}}{7}+\alpha_{4}^{2}\left(\frac{4222}{945}-\frac{64 \log (2)}{9}\right) \text {, } \\
& s_{10}\left(w_{1}\right)=\frac{1}{6300}\left(w_{1}^{2}+1\right)^{5}\left(7199-2520 \log (4)+315\left(16 \sinh ^{-1}\left(w_{1}\right)\right.\right. \\
& +\frac{1}{315}\left(-\frac{420\left(15 w_{1}^{4}+24 w_{1}^{2}+10\right)}{\left(w_{1}^{2}+1\right)^{3}}-\frac{1}{\left(w_{1}^{2}+1\right)^{9 / 2}} w_{1}\left(21000 w_{1}^{2}+5040\right.\right. \\
& \left.\left.+7199 w_{1}^{8}+24048 w_{1}^{6}+33264 w_{1}^{4}\right)-2520 \log \left(w_{1}^{2}+1\right)\right) \beta_{4} \beta_{6} \\
& +\frac{1}{18}\left(\beta_{10}-\beta_{4} \beta_{6}\right)\left(w_{1}^{2}+1\right)^{5}-\frac{1}{18}\left(\beta_{10}-\beta_{4} \beta_{6}\right) w_{1}^{9} \sqrt{w_{1}^{2}+1} \\
& \stackrel{w_{1} \rightarrow 0}{=} \frac{\beta_{10}}{18}-\frac{\beta_{4} \beta_{6}(840 \log (4)-883)}{2100}=\frac{2 \alpha_{10}}{9}+\frac{4 \alpha_{4} \alpha_{6}(2999-2520 \log (4))}{1575} \text {. }
\end{aligned}
$$

These coefficients can be written in terms of the $\alpha$ 's appearing in (2.3) by means of the following relations:

$$
\begin{aligned}
& \beta_{2}=4 \alpha_{2}, \quad \beta_{4}=4 \alpha_{4}+8 \alpha_{2}^{2}, \quad \beta_{6}=4 \alpha_{6}+16 \alpha_{4} \alpha_{2}+\frac{32}{3} \alpha_{2}^{3}, \\
& \beta_{8} \stackrel{\alpha_{2} \rightarrow 0}{=} 4 \alpha_{8}+8 \alpha_{4}^{2}, \quad \beta_{10} \stackrel{\alpha_{2} \rightarrow 0}{=} 4 \alpha_{10}+16 \alpha_{4} \alpha_{6},
\end{aligned}
$$

which can be obtained by equating the coefficients in the series expansion of (4.16).

\section{APPENDIX B: THE DIFFERENCE BETWEEN THE $z_{1}$-REGULARIZATION AND THE $\epsilon$-REGULARIZATION}

In terms of the variables,

$$
t=\sqrt{1+\left(\frac{z_{1}}{a}\right)^{2}-\left(\frac{r}{a}\right)^{2}}, \quad \psi(t)=\left(\frac{z}{a}\right)^{2},
$$

the $\mathrm{NG}$ action is

$$
S_{\mathrm{NG}}=\frac{L^{2}}{\alpha^{\prime}} \int_{w_{1}} \frac{\sqrt{1+w_{1}^{2}}}{e^{2\left(a^{2} \alpha_{2} \psi+a^{4} \alpha_{4} \psi^{2}\right)} t \sqrt{4+\frac{\left(1+w_{1}^{2}-t^{2}\right) \psi^{\prime}(t)^{2}}{t^{2} \psi(t)}}} d t
$$

The solution to the equation of motion with the boundary conditions,

$$
\psi\left(w_{1}\right)=w_{1}^{2} \equiv\left(z(a)=z_{1}\right), \quad \psi^{\prime}\left(\sqrt{1+w_{1}^{2}}\right)=0, \quad \equiv\left(z^{\prime}(0)=0\right),
$$

up to order $a^{2}$ is given by 


$$
\begin{aligned}
\psi(t)= & t^{2}-4 a^{2} \alpha_{2}\left(w_{1}^{2}+1\right)\left(\left(w_{1}^{2}+1\right) \log \left(-t^{2}+w_{1}^{2}+1\right)+\left(w_{1}-t\right)\left(-t+2 \sqrt{w_{1}^{2}+1}-w_{1}\right)\right. \\
& \left.+2\left(w_{1}^{2}+1\right) \tanh ^{-1}\left(\frac{t}{\sqrt{w_{1}^{2}+1}}\right)-2\left(w_{1}^{2}+1\right) \sinh ^{-1}\left(w_{1}\right)\right)
\end{aligned}
$$

and replacing in (B1) gives the following expression for the integrand up to order $a^{2}$ :

$$
\begin{aligned}
I\left(w_{1}, t\right)= & \frac{\sqrt{1+w_{1}^{2}}}{t^{2}}+a^{2} \frac{2 \alpha_{2} \sqrt{w_{1}^{2}+1}}{t^{4}}\left(2 t^{4}+4 t^{2} w_{1}^{2}-2 t^{2} w_{1} \sqrt{w_{1}^{2}+1}-t^{2} w_{1}^{2} \log \left(-t^{2}+w_{1}^{2}+1\right)-t^{2} \log \left(-t^{2}+w_{1}^{2}+1\right)\right. \\
& +6 w_{1}^{2} \log \left(-t^{2}+w_{1}^{2}+1\right)+3 \log \left(-t^{2}+w_{1}^{2}+1\right)+2\left(w_{1}^{2}+1\right)\left(t^{2}-3\left(w_{1}^{2}+1\right)\right) \sinh ^{-1}\left(w_{1}\right) \\
& -2\left(w_{1}^{2}+1\right)\left(t^{2}-3 w_{1}^{2}-3\right) \tanh ^{-1}\left(\frac{t}{\sqrt{w_{1}^{2}+1}}\right)+3 w_{1}^{4} \log \left(-t^{2}+w_{1}^{2}+1\right) \\
& \left.+3 t^{2}-6 t w_{1}^{2} \sqrt{w_{1}^{2}+1}-6 t \sqrt{w_{1}^{2}+1}-3 w_{1}^{4}-3 w_{1}^{2}+6 w_{1} \sqrt{w_{1}^{2}+1}+6 w_{1}^{3} \sqrt{w_{1}^{2}+1}\right) .
\end{aligned}
$$

The last term in this integrand is proportional to $w_{1}^{3} / t^{4}$, which vanish when $w_{1} \rightarrow 0$. However, integrating and then taking the limit, they lead to a nonvanishing result,

$$
\int_{w_{1}}^{\sqrt{1+w_{1}^{2}}} d t \frac{w_{1}^{3}}{t^{4}}=-\left.\frac{1}{3} \frac{w_{1}^{3}}{t^{3}}\right|_{w_{1}} ^{\sqrt{1+w_{1}^{2}}} \stackrel{w_{1} \rightarrow 0}{=} \frac{1}{3} .
$$

[1] G. 't Hooft, A planar diagram theory for strong interactions, Nucl. Phys. B72, 461 (1974).

[2] J. M. Maldacena, The large $N$ limit of superconformal field theories and supergravity, Adv. Theor. Math. Phys. 2, 231 (1998).

[3] E. Witten, Anti-de Sitter space and holography, Adv. Theor. Math. Phys. 2, 253 (1998).

[4] S. S. Gubser, I. R. Klebanov, and A. M. Polyakov, Gauge theory correlators from noncritical string theory, Phys. Lett. B 428, 105 (1998).

[5] O. Aharony, S. S. Gubser, J. M. Maldacena, H. Ooguri, and Y. Oz, Large $N$ field theories, string theory and gravity, Phys. Rep. 323, 183 (2000).

[6] S. S. Gubser, Dilaton driven confinement, arXiv:hep-th/ 9902155.

[7] T. Sakai and S. Sugimoto, Low energy hadron physics in holographic QCD, Prog. Theor. Phys. 113, 843 (2005).

[8] L. Da Rold and A. Pomarol, Chiral symmetry breaking from five dimensional spaces, Nucl. Phys. B721, 79 (2005).

[9] J. Erlich, E. Katz, D. T. Son, and M. A. Stephanov, QCD and a Holographic Model of Hadrons, Phys. Rev. Lett. 95, 261602 (2005).

[10] J. Polchinski and M. J. Strassler, The string dual of a confining four-dimensional gauge theory, arXiv:hep-th/0003136.

[11] C. Csaki and M. Reece, Toward a systematic holographic QCD: A braneless approach, J. High Energy Phys. 05 (2007) 062.
[12] J. M. Maldacena, Wilson Loops in Large $N$ Field Theories, Phys. Rev. Lett. 80, 4859 (1998).

[13] S. Rey and J. Yee, Macroscopic strings as heavy quarks in large $N$ gauge theory and anti-de Sitter supergravity, Eur. Phys. J. C 22, 379 (2001).

[14] M. A. Shifman, Wilson loop in vacuum fields, Nucl. Phys. B173, 13 (1980).

[15] O. Andreev and V. I. Zakharov, Gluon condensate, Wilson loops and gauge/string duality, Phys. Rev. D 76, 047705 (2007).

[16] R. Carcasses Quevedo, J. L. Goity, and R. Trinchero, QCD condensates and holographic Wilson loops for asymptotically AdS spaces, Phys. Rev. D 89, 036004 (2014).

[17] N. Drukker, D. J. Gross, and H. Ooguri, Wilson loops and minimal surfaces, Phys. Rev. D 60, 125006 (1999).

[18] C.-S. Chu and D. Giataganas, UV-divergences of Wilson loops for gauge/gravity duality, J. High Energy Phys. 12 (2008) 103.

[19] U. Gursoy and E. Kiritsis, Exploring improved holographic theories for QCD: Part I, J. High Energy Phys. 02 (2008) 032 .

[20] J. L. Goity and R. C. Trinchero, Holographic models and the QCD trace anomaly, Phys. Rev. D 86, 034033 (2012).

[21] Y. Kinar, E. Schreiber, and J. Sonnenschein, Q anti-Q potential from strings in curved space-time: Classical results, Nucl. Phys. B566, 103 (2000). 